\title{
Rhynchophylla total alkaloid rescues autophagy, decreases oxidative stress and improves endothelial vasodilation in spontaneous hypertensive rats
}

\author{
Chao $\mathrm{LI}^{1, \#}$, Feng JIANG ${ }^{2, \#}$, Yun-Iun $\mathrm{LI}^{1,2, *}$, Yue-hua JIANG ${ }^{2}$, Wen-qing YANG ${ }^{1}$, Jie SHENG ${ }^{1}$, Wen-juan XU ${ }^{3}$, Qing-jun ZHU ${ }^{1}$ \\ ${ }^{1}$ Shandong University of Traditional Chinese Medicine, Ji-nan 250000, China; ${ }^{2}$ Department of Cardiovascular, Affiliated Hospital of \\ Shandong University of Traditional Chinese Medicine, Ji-nan 250014, China; ${ }^{3}$ Binzhou Medical University, Yan-tai 264003, China
}

\begin{abstract}
Autophagy plays an important role in alleviating oxidative stress and stabilizing atherosclerotic plaques. However, the potential role of autophagy in endothelial vasodilation function has rarely been studied. This study aimed to investigate whether rhynchophylla total alkaloid (RTA) has a positive role in enhancing autophagy through decreasing oxidative stress, and improving endothelial vasodilation. In oxidized low-density lipoprotein (ox-LDL)-treated human umbilical vein endothelial cells (HUVECs), RTA (200 mg/L) significantly suppressed ox-LDL-induced oxidative stress through rescuing autophagy, and decreased cell apoptosis. In spontaneous hypertensive rats (SHR), administration of RTA (50 mg. $\mathrm{kg}^{-1} \cdot \mathrm{d}^{-1}$, ip, for 6 weeks) improved endothelin-dependent vasodilation of thoracic aorta rings. Furthermore, RTA administration significantly increased the antioxidant capacity and alleviated oxidative stress through enhancing autophagy in SHR. In ox-LDL-treated HUVECs, we found that the promotion of autophagy by RTA resulted in activation of the AMPactivated protein kinase (AMPK) signaling pathway. Our results show that RTA treatment rescues the ox-LDL-induced autophagy impairment in HUVECs and improves endothelium-dependent vasodilation function in SHR.
\end{abstract}

Keywords: hypertension; atherosclerosis; rhynchophylla total alkaloid; endothelial cells; autophagy; oxidized low-density lipoprotein; rapamycin; 3-MA; oxidative stress; endothelium-dependent vasodilation

Acta Pharmacologica Sinica (2018) 39: 345-356; doi: 10.1038/aps.2017.120; published online 09 Nov 2017

\section{Introduction}

Seeking antihypertensive drugs with vascular protective function is key to the treatment of hypertension. Inflammatory damage, oxidative stress and vascular endothelial dysfunction are considered the most important risk factors for vascular injury. Essential hypertension is a major contributor to the damage of vascular endothelial cells and the most relevant cardiovascular risk factor. Although the underlying mechanisms of hypertension are not yet fully clear, several studies have shown that oxidative stress is recognized as a central factor in pathophysiological hypertension. Moreover, oxidative stress and antioxidant system are ubiquitous aspects of animal models of hypertension ${ }^{[1,2]}$, and oxidative stress is increased in patients with essential hypertension ${ }^{[3,4]}$.

The disintegration of the balance between vasoconstriction and vasodilation contributes to the disruption of endothelial

\footnotetext{
\# These authors contributed equally to this work.

${ }^{*}$ To whom correspondence should be addressed.

E-mail yunlun.lee@hotmail.com

Received 2017-04-12 Accepted 2017-07-26
}

cells, and oxidative stress is considered to play a key role in this pathogenesis ${ }^{[5]}$. The injury of vascular endothelial cells plays a critical role in the occurrence and development of atherosclerosis. The function of vascular endothelial cells is impaired in essential hypertension patients for many reasons, among which oxidative stress is a crucial factor ${ }^{[6]}$. Some major enzyme systems produce reactive oxygen species (ROS), such as nicotinamide adenine dinucleotide phosphate (NADPH) oxidase and endothelial nitric oxide synthase (eNOS). NADPH oxidase is likely to be responsible for the increase in ROS in essential hypertension ${ }^{[7]}$. The insufficient nitric oxide (NO) bioavailability affected by eNOS may lead to endothelial dysfunction $^{[8]}$. Elevated levels of ROS can decrease NO levels. NO instability enhances the production of superoxide anion, which in turn reacts with $\mathrm{NO}$ and produces peroxynitrite, thus further resulting in destruction of eNOS and generation of more superoxide ${ }^{[9]}$.

Oxidation of LDL (ox-LDL) is an important risk factor in the atherogenic progression that leads to endothelial activation and injury, thus resulting in an inflammatory response ${ }^{[10]}$. Many inflammation markers are highly expressed in hypertensive 
patients ${ }^{[11]}$. Thus, protecting vascular endothelial cells from ox-LDL damage might be a focus for atherogenesis prevention in essential hypertension therapy.

Autophagy is a protective mechanism that defends plaque cells and endothelial cells against some stimulation in the arterial wall, especially oxidative injury ${ }^{[12,13]}$. Moreover, autophagy increases NO bioavailability and decreases vascular inflammation, thereby decreasing oxidative stress and preserving NO-mediated arterial endothelium-dependent dilatation ${ }^{[14]}$. Two major signaling pathways, class I phosphatidylinositol-3 kinase (class I PI3K)/protein kinase B (PKB, also known as Akt)/mammalian target of rapamycin (mTOR) and adenosine monophosphate-activated protein kinase (AMPK)/sirtuin 1 (SIRT1), contribute to regulate the formation of autophagosomes ${ }^{[15,16]}$. In recent years, studies have increasingly focused on activating autophagy through interfering with those pathways to decrease ox-LDL injury.

The rhynchophylla total alkaloid (RTA) is the major active ingredient of Uncaria. We have previously shown that RTA has a strong antihypertensive effect and a protective effect on human umbilical vein endothelial cells (HUVECs). Further studies have demonstrated that RTA inhibits the secretion of intercellular adhesion molecule-1 (ICAM-1) and vascular cell adhesion molecule-1 (VCAM-1) through decreasing the inflammatory reaction in vessel walls ${ }^{[17,18]}$. This study aimed to investigate whether RTA has a positive role in enhancing autophagy, thereby decreasing oxidative stress and improving endothelial vasodilation.

\section{Materials and methods}

The ethics committee of Shandong University of Chinese Traditional Medicine approved this study.

\section{Antibodies}

Antibodies against Beclin-1 (D40C5), LC3A/B (D3U4C), and eNOS (49G3) were from Cell Signaling Technology (Danvers, MA, USA); anti-p62 antibody (ab91526), anti-MAP1LC3B antibody (ab48394), and Anti-NADPH oxidase 4 antibody (ab133303) were from Abcam (Burlingame, CA, USA); pEGFPLC3 was from Biovector Co, Ltd (Beijing, China); horseradish enzyme mark goat rabbit IgG was from Zhongshan Golden Bridge Biotechnology (Beijing, China); $\beta$-actin was from Nanjing Jiancheng Biological Engineering Institute (Nanjing, China).

\section{The preparation of RTA}

RTA was provided by Prof Hong-lei ZHOU of the Shandong University of Traditional Chinese Medicine. Briefly, RTA was extracted by ethanol in Uncaria. The extraction time was $2 h$, and three extractions were performed with $75 \%$ ethanol. The purification process was performed with a macroporous cation exchange resin 732 column at a $1 \mathrm{~mL} \cdot \mathrm{min}^{-1}$ flow rate. 10 BV water and 6 BV $80 \%$ ethanol, respectively, were used to remove impurities. Finally, $1 \%$ ammonium and $80 \%$ ethanol were used for elution. RTA was dissolved in DMSO and then diluted to the working concentration. The content of DMSO was $4 \%$. The solution was stored at $4{ }^{\circ} \mathrm{C}$.

\section{Cell culture and drug treatment}

HUVECs were provided by the Central Laboratory of the Shandong University of Traditional Chinese Medicine. HUVECs were cultured in endothelial cell medium (ECM) containing 5\% FBS, 1\% ECGS and 1\% P/S Solution (ScienCell, California, USA). Cells were maintained at $37^{\circ} \mathrm{C}$ and $5 \% \mathrm{CO}_{2}$ in a humidified incubator. HUVECs were incubated with 100 $\mu \mathrm{g} \cdot \mathrm{mL}^{-1}$ ox-LDL (Solarbio, Beijing, China) as a control. Except for the normal control group (cultured only with ECM), other groups were cultured with drugs for $1 \mathrm{~h}$ before adding oxLDL. All cells were incubated for an additional $24 \mathrm{~h}$ before analysis, in accordance with our previous study.

\section{Animals}

7-week-old male spontaneously hypertensive rats (SHR) and Wistar-Kyoto (WKY) rats were obtained from Vital River Laboratory Animal Technology Co (Beijing, China). SHR were randomly assigned to 4 groups, and the WKY rats were assigned to normal groups ( $\mathrm{n}=8$ rats per group). (1) WKY group: $0.9 \% \mathrm{Nacl}\left(2 \mathrm{~mL} \cdot \mathrm{kg}^{-1}\right)$; (2) SHR group: $0.9 \% \mathrm{Nacl}(2$ $\left.\mathrm{mL} \cdot \mathrm{kg}^{-1}\right)$; (3) Rap group: Rap (1 mg. $\left.\mathrm{kg}^{-1}\right)$; (4) RTA group: RTA (50 mg. $\left.\mathrm{kg}^{-1}\right)$; (5) RTA+3-MA group: RTA $\left(50 \mathrm{mg} \cdot \mathrm{kg}^{-1}\right)$ and 3-MA (15 mg. $\left.\mathrm{kg}^{-1}\right)$. All groups were administered drugs by intraperitoneal injection for six weeks. We regularly checked the rat weight in the 5 groups every week and adjusted the dosage according to the weight of the rats. Animal care and procedures conformed to the Guide for the Care and Use of Laboratory Animals.

\section{The plasma concentration and metabolism of RTA}

Briefly, plasma was separated from blood by centrifugation at $2220 \times g$ for $15 \mathrm{~min}$. Methanol $(500 \mu \mathrm{L})$ was added to the plasma samples $(100 \mu \mathrm{L})$ and then analyzed by liquid chromatography-electrospray ionization Q-Exactive. Separations were performed on Halo C18 columns $(2.1 \times 100 \mathrm{~mm}, 2.7 \mu \mathrm{m}$, advanced materials technology). The electrospray ionization mass spectrometry spectra were acquired in positive ionization mode $\left(\mathrm{m} / \mathrm{z}: 385.211[\mathrm{M}+\mathrm{H}]^{+}\right)$.

\section{Western blot analysis}

HUVECs were washed with cold phosphate-buffered saline (PBS) after being incubated and lysed with cell lysis buffer (Beijing Solarbio Science \& Technology Co, Ltd, Beijing, China). Protein concentrations were measured using a BCA protein assay kit (Beyotime Institute of Biotechnology, Shanghai, China). Protein samples were separated by sodium dodecyl sulfate-polyacrylamide gel electrophoresis (SDS-PAGE) and then transferred to polyvinylidenedifluoride (PVDF) membranes (Beijing Solarbio Science \& Technology Co, Ltd, Beijing, China) by electroblotting. After being blocked with $5 \%$ skim milk for one hour, the membranes were incubated with primary antibodies (1:1000) overnight at $4{ }^{\circ} \mathrm{C}$, and then incubated with horseradish peroxidase-conjugated secondary antibodies (Zhongshan Golden Bridge Biotechnology Co, Beijing, China)(1:20000). After one hour, bands were detected with Immobilon Western HRP Substrate (Millipore 
Corporation, Billerica, USA), and the average densitometric analysis was conducted using Image-Pro Plus 4.5 software.

\section{MAP1LC3B immunofluorescence microscopy}

Cells or tissue sections were rinsed in phosphate-buffered saline, fixed in $4 \%$ paraformaldehyde at room temperature for $20 \mathrm{~min}$, and then blocked with immunol staining blocking buffer for $1 \mathrm{~h}$ at $4^{\circ} \mathrm{C}$. MAP1LC3B antibody (1:50) was added and incubated overnight at $4^{\circ} \mathrm{C}$. After being washed with phosphate buffered saline, cells were incubated with the appropriate secondary antibody (1:100) for $1 \mathrm{~h}$ at room temperature, and the nuclei were stained with DAPI (Beyotime Biotechnology, Shanghai, China). Thereafter, cells were observed by laser-scanning microscopy (Zeiss Vert.A1, Carl Zeiss Canada).

\section{Transfection of GFP-LC3 and si RNA}

Cells were seeded on cover slips in 24-well plates. The next day, according to the manufacturer's instructions, we used Lipofectamine 2000 in OPTI-MEM medium to transiently transfect the $0.8 \mathrm{mg}$ plasmid/well GFP-LC3 expressing plasmids into the cells in $37^{\circ} \mathrm{C}$ for $6 \mathrm{~h}$, and then, cells were cultured with ECM medium (containing 5\% FBS) for $24 \mathrm{~h}$. The transfection efficiency of GFP-LC3 was $65 \%$. Cover slips were mounted on glass slides, and cells were observed by fluorescence confocal microscopy. We counted the number of GFPLC3 punctuated dots on a per-cell basis in at least 3 different trials ${ }^{[50]}$. Adenovirus vector was used for si RNA transfection. According to the manufacturer's protocol (Han Biological Technology Co, Ltd, Shanghai, China), briefly, after cells reached $50 \%-70 \%$ confluence, adenovirus vector was added according to the adenovirus small volume infection table (MOI=100). After transfection for $48 \mathrm{~h}$, the expression level of beclin-1 in adenovirus-transduced cells was detected to confirm successful knockdown.

\section{Apoptosis measurement (Annexin-V/ PI double staining)}

Apoptosis was determined by flow cytometry using an Apoptosis Detection Kit (Annexin-V/PI double staining). Briefly, according to the manufacturer's instructions, after different treatments, cells were digested with pancreatic enzyme (excluding EDTA). We collected and washed cells and then added 400 $\mu \mathrm{L} 1 \times$ Annexin $\mathrm{V}$ binding buffer to keep the cells in suspension. Next, cells were incubated with Annexin V-FITC staining fluid for $15 \mathrm{~min}$ at $4^{\circ} \mathrm{C}$ in a dark environment, and then, PI staining solution was added and incubated for $5 \mathrm{~min}$ at $4^{\circ} \mathrm{C}$. The apoptosis rate of HUVECs was detected by flow cytometry.

\section{Transmission electron microscopy (TEM)}

The autophagosomes were observed by TEM. After collection, cells formed a dense cell mass and were then fixed with $3 \%$ glutaraldehyde fixation fluid ( $\mathrm{pH} 7.2-7.4$ ), According to the conventional TEM sample preparation method, cells were rinsed, fixed in 1\% osmic acid, rinsed, dehydrated, saturated, and embedded in Epon812; Semithin sections were cut with an LKB-V ultra-thin slicing machine, electronically dyed with lead citrate and uranium acetate, and examined with a JEOL-
1200EX transmission electron microscope.

\section{Vascular ring tension test}

Isolated rat thoracic aortas were maintained in $5.0 \mathrm{~mL}$ of Krebs solution (composition in mmol/L: $\mathrm{NaCl} 118, \mathrm{KCl} 4.75$, $\mathrm{NaHCO}_{3} 25, \mathrm{MgSO}_{4} 1.2, \mathrm{CaCl}_{2} 2, \mathrm{KH}_{2} \mathrm{PO}_{4} 1.2$ and glucose 11, $\mathrm{pH} 7.4$,) and gassed with $95 \% \mathrm{O}_{2}$ and $5 \% \mathrm{CO}_{2}$ at $37^{\circ} \mathrm{C}$. After being cleaned up under a microscope and clipped into $4 \mathrm{~mm}$ long aortic rings, aortic rings were connected to hooks and maintained with $1 \mathrm{~g}$ resting tension for $60 \mathrm{~min}$. Then phenylephrine $\left(0.1 \mathrm{mmol} \cdot \mathrm{L}^{-1}\right)$ in $5 \mathrm{~mL}$ of Krebs solution was added, and samples were incubated until vascular tension stability was achieved; then, we added acetylcholine (ACh) $\left(10^{-9}-10^{-5} \mathrm{~mol} \cdot \mathrm{L}^{-1}\right)$ and recorded the changes in the tension. Krebs solution was used to flush aortic rings to restore their initial tension state, and then, aortic contraction was excited by phenylephrine $\left(0.1 \mathrm{mmol} \cdot \mathrm{L}^{-1}\right)$. Until the maximum tension was reached, sodium nitroprusside (SNP) was gradually added to relax blood vessels.

\section{Superoxide dismutase (SOD) and microscale malondialdehyde (MDA) assay}

SOD activity in cell culture supernatants and ser were measured with an SOD assay kit (WST-1 method) (Nanjing Jiancheng Biological Engineering Institute, Nanjing, China). Briefly, according to the kit instructions, before formal detection, we selected two normal control-group samples to check as preliminary experiments, and the absorbance was measured at a wavelength of $450 \mathrm{~nm}$. MDA content analysis was performed with Microscale MDA assay kit (TBA method) (Nanjing Jiancheng Biological Engineering Institute, Nanjing, China). After treatment, cell culture supernatant samples (0.1 $\mathrm{ml}$ ) were detected in the 532-nm wavelength.

\section{Serum NO, T-AOC and GSH-PX assay}

Blood samples were taken from the inferior vena cava. After standing for $30 \mathrm{~min}$, blood was centrifuged to obtain serum. The level of NO in serum was determined with an NO assay kit (Nitrate reductase method) (Nanjing Jiancheng Biological Engineering Institute, Nanjing, China). Briefly, referring to the kit instructions, reagent was added sequentially, and each step was performed in accordance with the requirements. After centrifugation, the supernatant was collected for coloration. Finally, the absorbance was measured at a 550-nm wavelength. Total antioxidant capacity (T-AOC) and GSH-PX in serum were determined with a T-AOC assay kit (Nanjing Jiancheng Biological Engineering Institute, Nanjing, China) and a GSH-PX assay kit (Nanjing Jiancheng Biological Engineering Institute, Nanjing, China). All steps were performed in accordance with the kit instructions, and the absorbances were measured at 520-nm and 412-nm wavelengths.

\section{Serum ALT, AST and creatinine assay}

ALT, AST and creatinine diagnostic kits were obtained from Sysmex Corporation (Kobe, Japan). All steps were performed in accordance with the manufacturer's instructions for diagnostic kits. The levels of ALT, AST and creatinine in the serum 
were determined with a CHEMIX-180 automatic biochemistry analyzer (Sysmex Corporation).

\section{Statistical analysis}

Data from at least three independent experiments are presented as the mean $\pm S D$. Two-group comparison was performed using a $t$-test for independent samples, whereas oneway ANOVA with Bonferroni's multiple comparison post hoc test was used for multiple-group statistical analyses. Raw data were analyzed with SPSS 17.0 software (SPSS, Inc, Chicago, IL, USA), and images were processed with Graphpad Prism 6 (GraphPad Software, La Jolla, CA, USA). $P$-Values $<0.05$ were considered statistically significant.

\section{Results}

Autophagy protected against apoptosis in ox-LDL-treated HUVECs To address the role of autophagy in ox-LDL-treated HUVECs, first, we detected the changes of autophagy in ox-LDL-treated HUVECs. As shown in Figures 1A-C, ox-LDL decreased the levels of LC3-II and beclin-1 in a time-dependent manner in HUVECs, and the effect was pronounced $24 \mathrm{~h}$ after ox-LDL exposure. In addition, the accumulation of p62 was markedly increased at $24 \mathrm{~h}$ after ox-LDL exposure. Rapamycin (Rap, an inhibitor of mTOR) and 3-methyladenine (3-MA, an inhibitor of autophagy) were used to activate and inhibit autophagy separately. The impaired autophagy in ox-LDL-treated HUVECs was rescued by Rap but aggravated by 3-MA (Figure 1D). The number of MAP1LC3B and GFP-LC3 dots also demonstrated a change in autophagy level (Figure 1e). In the next experiment, the apoptosis rate was detected to demonstrate the role of autophagy in ox-LDL-treated HUVECs. As shown in Figure 1f, the apoptosis rate was clearly increased after ox-LDL treatment for $24 \mathrm{~h}$, and this injury was rescued by Rap but exacerbated by 3-MA. These data suggested that Rap protects against apoptosis in ox-LDL-treated HUVECs via enhancing autophagy, and the opposite effect is exerted by 3-MA, which exacerbates the impaired autophagy induced by ox-LDL.

\section{RTA protected HUVECs from oxidative damage through rescuing the impairment of autophagy in ox-LDL-treated HUVECs}

Our previous studies have demonstrated that RTA has a protective effect on vascular endothelial cells in SHR. Therefore, we investigated the effect of RTA on autophagy in oxLDL-treated HUVECs. The impairment in autophagy was rescued by RTA, as demonstrated by the increased LC3II content, Beclin-1 level and the GFP-LC3 dots (Figures 2A and 2F). Autophagosomes were also observed by transmission electron microscopy (TEM); we found fewer autophagosomes and more lipid droplets after ox-LDL exposure, and this phenomenon was reversed by RTA. Furthermore, 3-MA played an opposite role that counteracted the effects of RTA (Figure 2E). We also performed apoptosis assays by double staining with Annexin V/PI to indicate the protective role of RTA in ox-LDL-stimulated HUVECs. RTA protected HUVECs from oxidative damage induced by ox-LDL, but that protection was abolished by 3-MA (Figure 2B). As shown in Figures 2C and $\mathrm{D}$, RTA rescued the impaired antioxidant capacity manifested by the increased superoxide dismutase (SOD) activity and lower microscale malondialdehyde (MDA) content. To attain more convincing results, we used beclin- 1 small interfering RNA (si RNA) to knock down beclin-1. In HUVECs transfected with con si RNA, RTA significantly rescued autophagy, as manifested by increased beclin- 1 and LC3-II/ $\beta$-actin ratios, and inhibited ox-LDL-induced cell apoptosis. RTA alleviated oxidative damage via rescuing the impaired autophagy manifested by the increased SOD activity and lower MDA content. In contrast, this result was not observed in Beclin-1 knockdown HUVECs (Supplementary Figure 1). Together, these results showed that RTA alleviates oxidative damage via rescuing the impaired autophagy in ox-LDL-treated HUVECs.

RTA improved endothelium-dependent vasodilation through enhancing autophagy in SHR

An increasing body of evidence indicates that autophagy can be activated by oxidative stress and further degrades oxidative damage ${ }^{[19,20]}$. However, long-term oxidative stress can impair autophagy. It has been reported that oxidative stress is ubiquitous in animal models of hypertension and plays a primary role in endothelial dysfunction ${ }^{[1,2,21]}$. Because RTA mitigated oxidative damage by enhancing autophagy, we further investigated whether RTA might improve endothelium-dependent vasodilation function through autophagy induction in SHR. We tested the toxic reactions of RTA and found no significant fluctuation in body weight or food and water intake in rats (Supplementary Figures 2A-C). Serum alanine transaminase (ALT), aspartate aminotransferase (AST) and creatinine also showed no impairment in the liver and kidney (Supplementary Figures 2D-E). As shown in Figure 3A, the endotheliumdependent vasodilation function stimulated by ACh was markedly decreased in SHR. In contrast, RTA and Rap clearly improved the endothelium-dependent vasodilation function, whereas 3-MA inhibited this effect. However, we also found that each group of thoracic aorta relaxation activity stimulated by sodium nitroprusside (SNP) had a good reaction in the endothelium-independent dilation test. Furthermore, we evaluated the serum levels of NO and the expression of eNOS in the thoracic aorta. The data indicated that RTA markedly promoted the production of NO (Figure $3 \mathrm{~B}$ ) and the level of eNOS (Figure 3C), both of which were impaired in SHR. As expected, this result was not observed in the group treated with 3-MA and RTA. Moreover, autophagy was markedly enhanced by RTA, as indicated by the increased levels of LC3II and beclin-1 (Figure 3D). The number of MAP1LC3B dots in the endothelium of the thoracic aorta also demonstrates the effect of RTA (Figure 3E). These data suggested that RTA improves endothelium-dependent vasodilation function through enhancing autophagy in SHR.

RTA strengthened antioxidant capacity and alleviated oxidative damage through enhancing autophagy in SHR

Owing to the close relationship between endothelium-depen- 
A
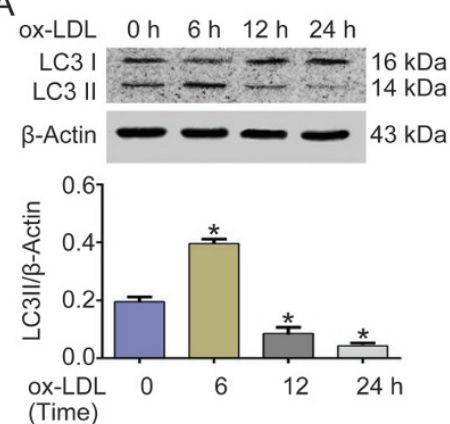

D

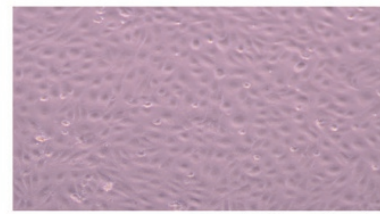

exposure to ox-LDL $(100 \mu \mathrm{g} / \mathrm{mL})$ for $24 \mathrm{~h}$

E

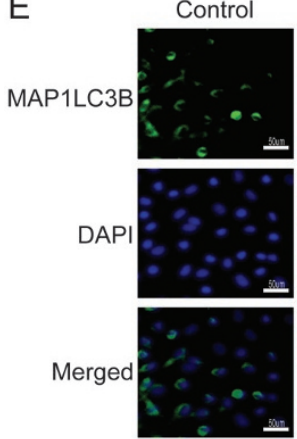

Control
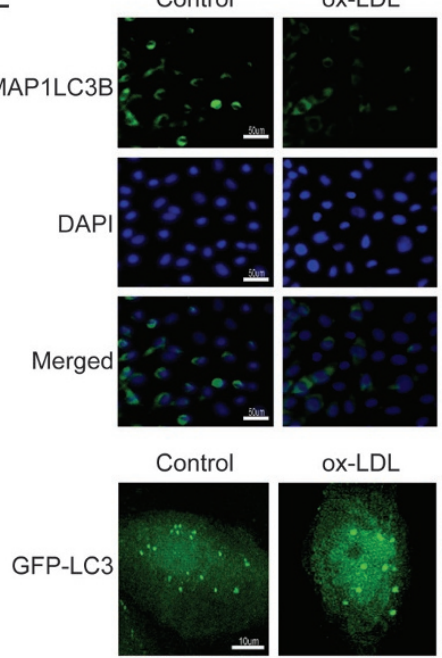

ox-LDL

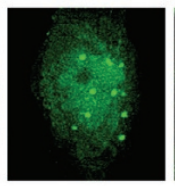

B
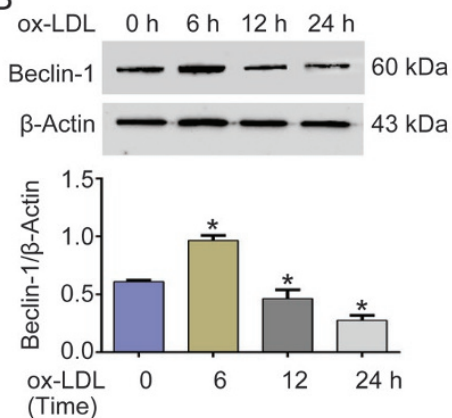

C
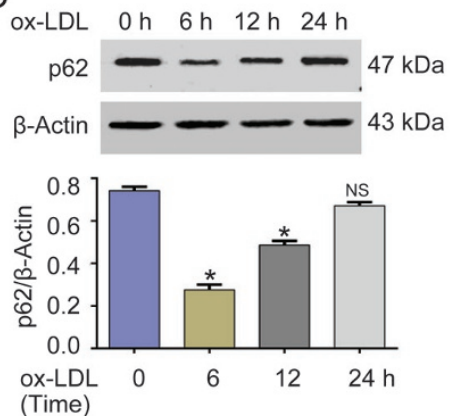
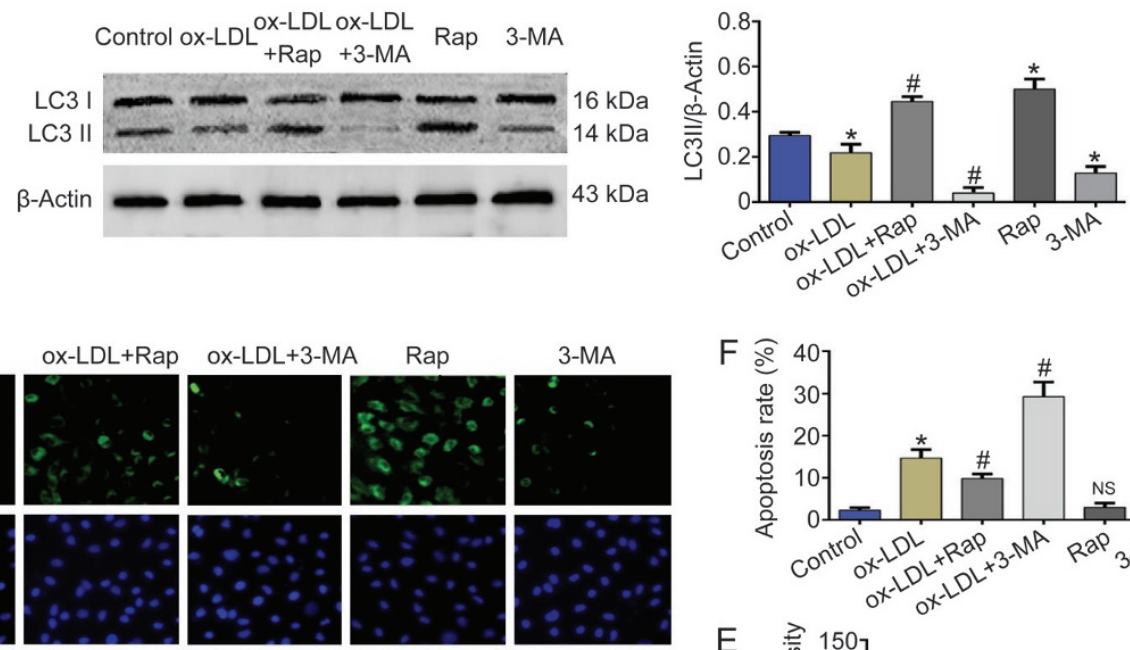

3-MA
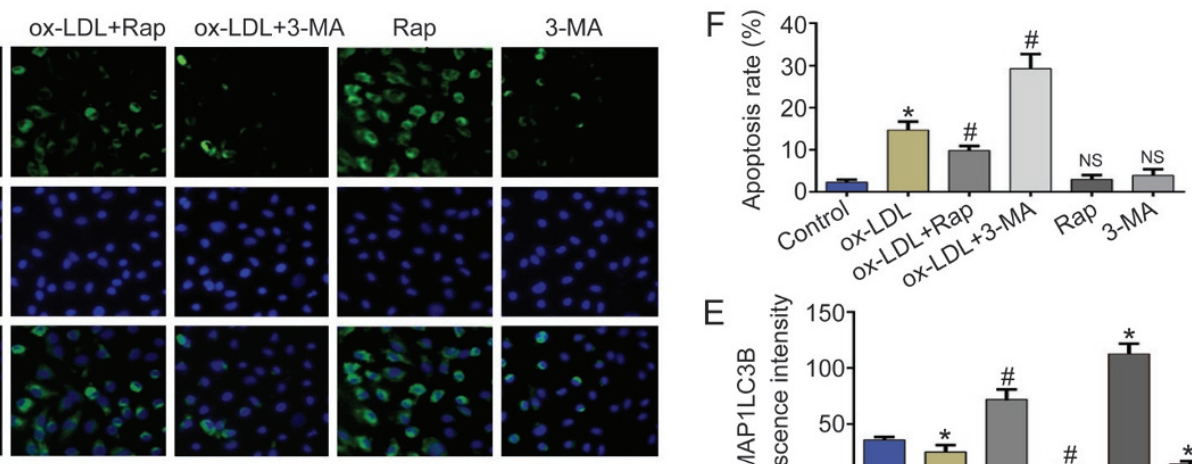

ox-LDL+Rap ox-LDL+3-MA
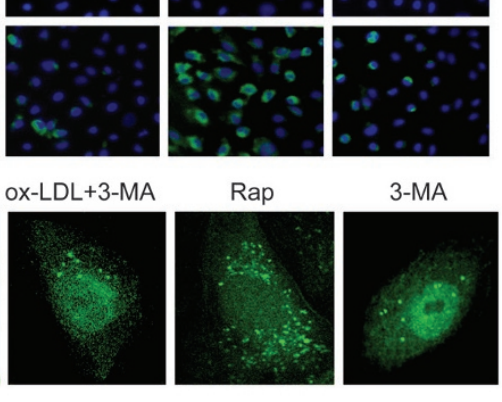

E
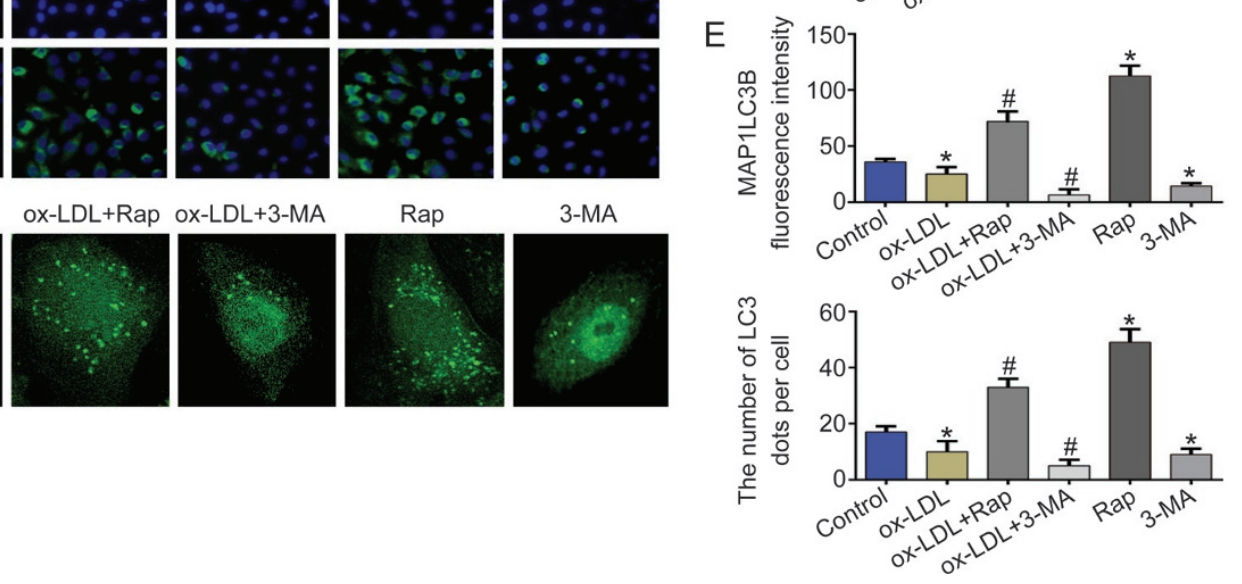

Figure 1. Autophagy protected against apoptosis in ox-LDL-treated HUVECs. Autophagy was obviously impaired by ox-LDL (100 $\mu g / m L)$ in a timedependent manner in HUVECs, and an obvious effect occurred at $24 \mathrm{~h}$ after ox-LDL exposure, which was manifested by the decreased LC3II content (A), beclin-1 level (B), and the increased p62 level (C). (D-E) HUVECs were incubated with either the culture medium (control) or ox-LDL (100 $\mu g / m L)$ in the absence or presence of rapamycin (Rap; $100 \mathrm{nmol} / \mathrm{L}$ ) and 3-methyladenine (3-MA; $5 \mathrm{mmol} / \mathrm{L}$ ) for $24 \mathrm{~h}$. It was shown that the impaired autophagy in oxLDL-treated HUVECs was rescued by Rap, which was aggravated by 3-MA. (F) Rap rescued the apoptosis induced by ox-LDL, whereas 3-MA exacerbated. ${ }^{\text {NS }} P>0.05$ vs control group, ${ }^{*} P<0.05$ vs control group; ${ }^{\#} P<0.05$ vs ox-LDL group. Data were pooled as mean \pm SD (error bars) from three independent experiments.

dent vasodilation function and oxidative stress, we analyzed the antioxidant ability and oxidative damage in SHR. As shown in Figure 4A, RTA clearly increased the total antioxidant capacity (T-AOC) in SHR, and this effect was inhibited by 3-MA. Furthermore, the decreased SOD activity and glutathione peroxidase (GSH-PX) levels, as well as greater MDA content in SHR, were reversed by RTA treatment (Figures
4B-D). Rap had a similar effect to RTA in increasing the antioxidant capacity and mitigating oxidative stress. A decreased production of $\mathrm{NO}$ or an increased production of ROS leads to an imbalance that promotes endothelial dysfunction. The most important source of ROS in the vasculature is NADPH oxidase $^{[22]}$. In our study, we separately analyzed the expression of NADPH oxidase 4 (Nox4) in the thoracic aorta and 

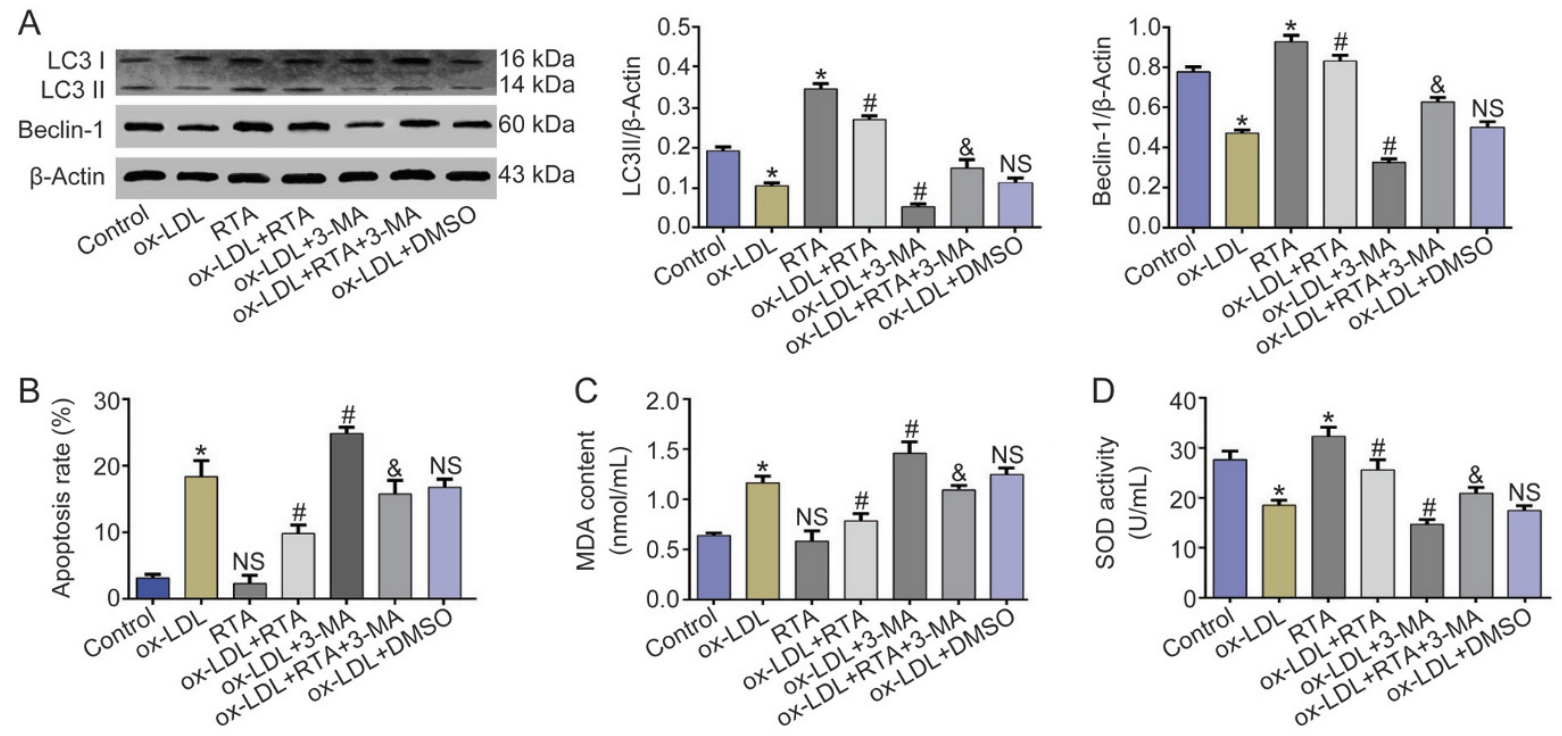

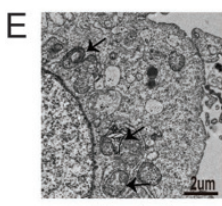

Control

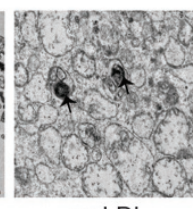

ox-LDL

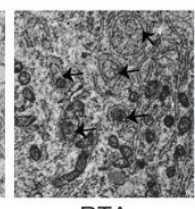

RTA

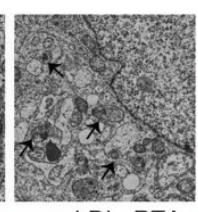

ox-LDL+RTA

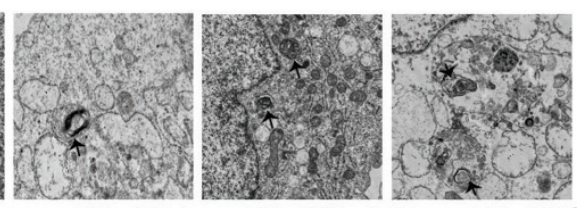

ox-LDL+3-MA ox-LDL+RTA+3-MA ox-LDL+DMSO

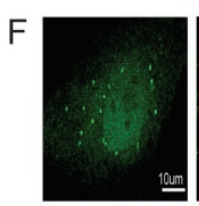

Control

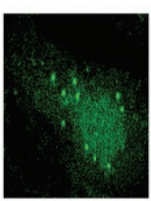

ox-LDL

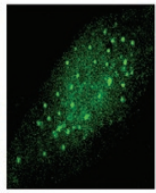

RTA

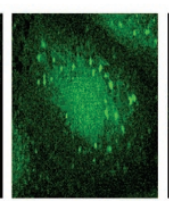

ox-LDL+RTA ox-LDL+3-MA
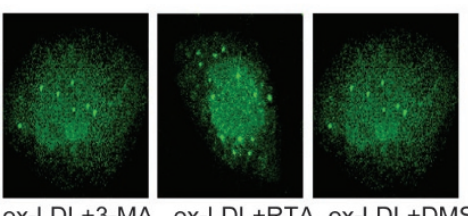

ox-LDL+RTA ox-LDL+DMSO $+3-\mathrm{MA}$

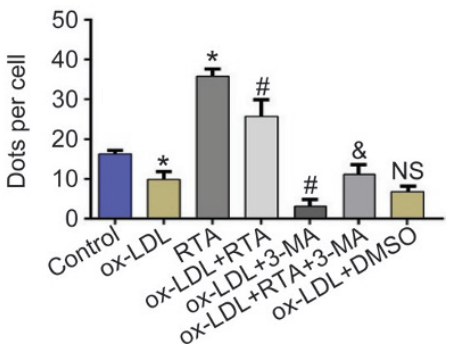

Figure 2. RTA protected HUVECs from oxidative damage through enhancing autophagy in ox-LDL-treated HUVECs. (A-F) HUVECs were incubated with either the culture medium (control) or ox-LDL $(100 \mu \mathrm{g} / \mathrm{mL}$ ) in the absence or presence of 3-MA (5 mmol/L), RTA (200 mg/L) and DMSO (4\%o) for $24 \mathrm{~h}$. RTA increased the level of LC3II and beclin-1 (A), the amount of autophagosomes (E) and the number of GFP-LC3 dots (F), that were abolished by 3-MA. (B) RTA rescued the apoptosis induced by ox-LDL, and 3-MA dramatically inhibited the effect of RTA. RTA rescued the impaired antioxidant capacity manifested by the increased of SOD activity (D) and less MDA content (C), whereas 3-MA abolished the effect of RTA. ${ }^{\text {Ns }} P>0.05$ vs control group, ${ }^{*} P<0.05$ vs control group; ${ }^{\text {NS }} P>0.05$ vs ox-LDL group, ${ }^{\#} P<0.05$ vs ox-LDL group; ${ }^{\circledR} P<0.05$ vs ox-LDL+RTA group. Data were pooled as mean \pm SD. (error bars) from three independent experiments.

HUVECs. RTA clearly decreased the expression of Nox4 in the thoracic aorta, but this effect was counteracted by 3-MA (Figure 4E). In ox-LDL-treated HUVECs, a similar result was found (Figure 4E). These data suggested that RTA strengthens antioxidant capacity and mitigates oxidative damage through enhancing autophagy.

Promotion of autophagy by RTA was relevant to activating the AMPK signaling pathway

To further investigate the signaling mechanisms of RTA in rescuing autophagy in ox-LDL-treated HUVECs, we detected the activation status of AMPK signaling. RTA markedly increased p-AMPK expression in ox-LDL-treated HUVECs (Figure 5A). Compound C, an AMPK inhibitor, was used to identify whether the AMPK signaling pathway was involved in the promotion of autophagy by RTA. As shown in Figures 5B-D, compound $\mathrm{C}$ reversed RTA's effects in increasing LC3II content, beclin-1 level and GFP-LC3 dots. These data suggested that the AMPK signaling pathway is involved in the promotion of autophagy by RTA.

\section{Discussion}

Vascular endothelial dysfunction is a crucial factor in the occurrence of atherosclerosis caused by hypertension, and oxidative stress is considered to be its major inducer ${ }^{[21,23]}$. Therefore, protecting vascular endothelial cells against oxidative stress injury can effectively delay the occurrence and development of atherosclerosis in patients with hypertension. Despite extensive reports indicating that oxidative stress contributes to endothelial dysfunction and atherogenesis, antioxidant treat- 

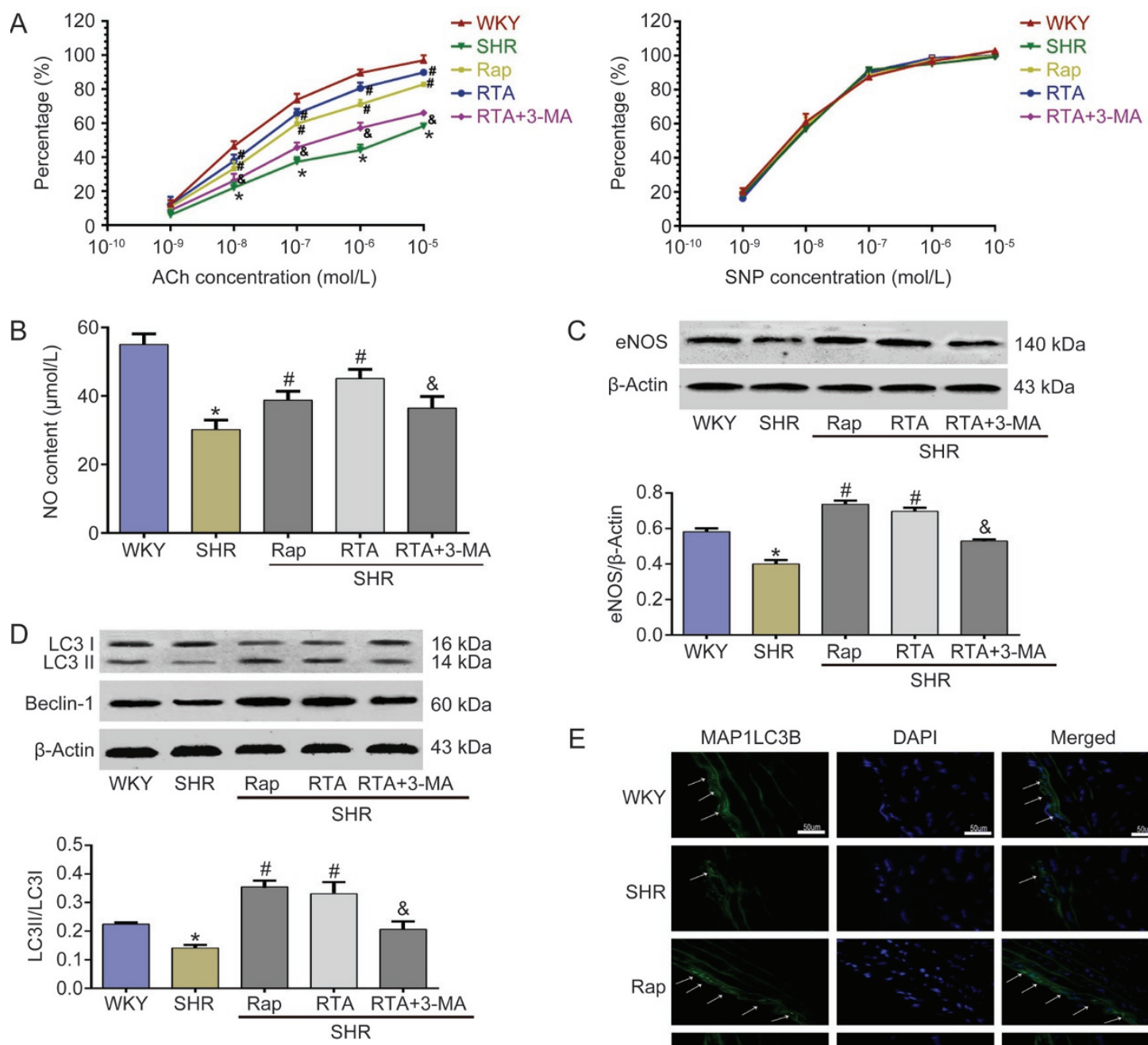

$\mathrm{E}$
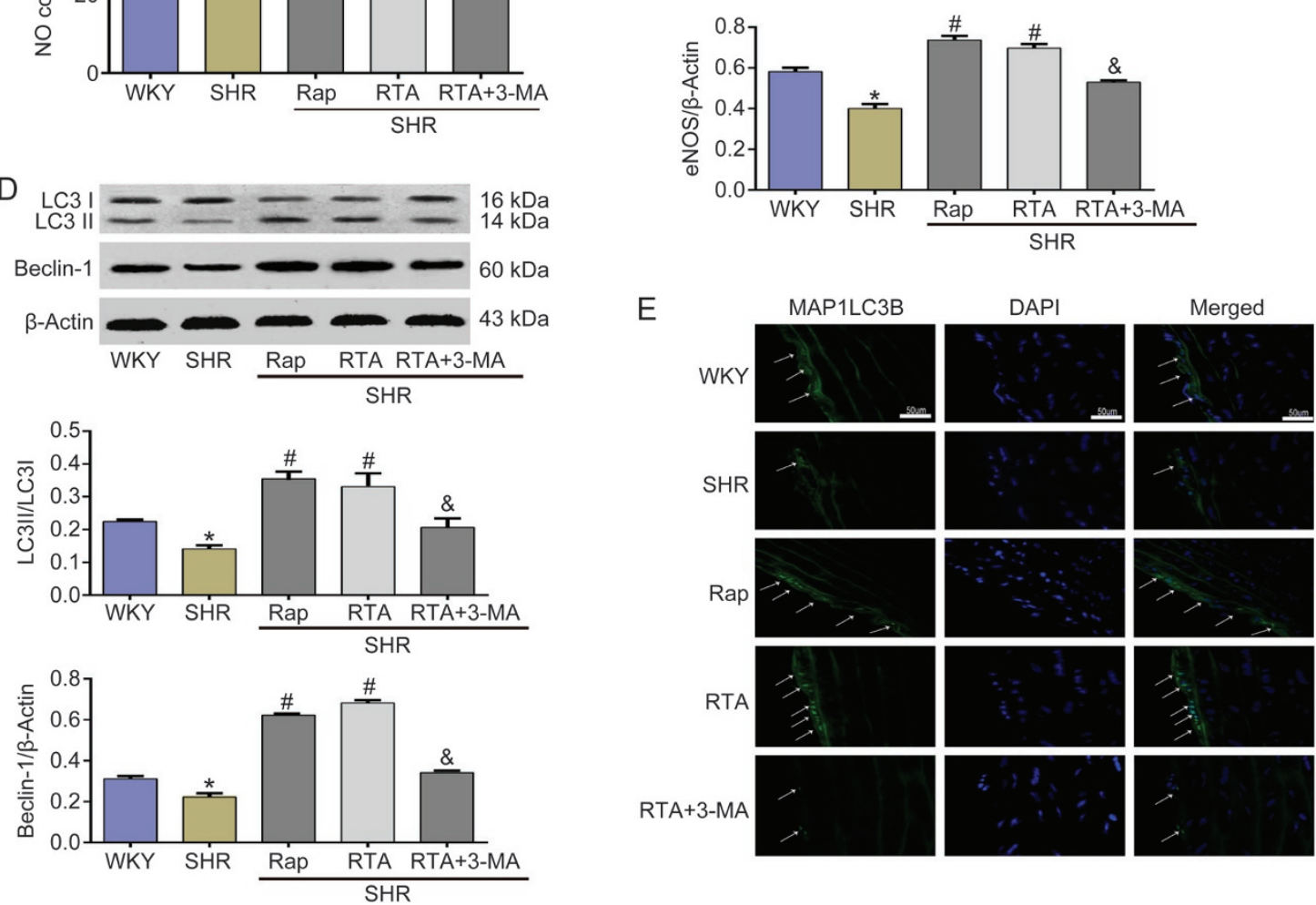

Figure 3. RTA improved endothelium-dependent vasodilation function through enhancing autophagy in SHR. (A) RTA improved endothelium-dependent vasodilation function. In contrast, RTA exerted no effect on the endothelium- independent vasodilation function. RTA increased the expression of eNOS (C) in thoracic aorta and NO content (B) in SHR serum, and the effect was abolished by 3-MA which inhibited the autophagy. (D) RTA obviously increased the expression of LC3-II and Beclin-1 in thoracic aorta isolated from SHR. (E) The number of MAP1LC3B dots in the endothelium of the thoracic aorta were increased in RTA group, whereas 3-MA abolished the effect of RTA. ${ }^{*} P<0.05$ vs WKY, ${ }^{~} P<0.05$ vs SHR, ${ }^{\circledR} P<0.05$ vs RTA group. Data were pooled as mean $\pm S D$ (error bars) from three independent experiments.

ment has failed to show a significant benefit. Our study demonstrated that autophagy protected against apoptosis in oxLDL-treated HUVECs. However, long-term oxidative stress can damage autophagy; RTA had a positive role in enhancing autophagy and thus decreasing ox-LDL-induced oxidative damage and improved endothelial vasodilation; RTA improved the antioxidant capacity and alleviated oxidative damage through enhancing autophagy in SHR. In addition, promotion of autophagy by RTA resulted in activation of the AMPK signaling pathway.
Autophagy is a dynamic and highly conserved regulated cellular catabolic process that delivers long-lived proteins and organelles into lysosomes for degradation and recycling ${ }^{[2,25]}$. LC3, a ubiquitin-like protein, is an excellent marker for monitoring autophagy. LC3 is classified into two forms, nonlipidated and lipidated, which are referred to as LC3-I and LC3II, respectively. However, the total amount of LC3 is not necessarily increased in mammalian cells when autophagy is induced, because there is a possibility of conversion of LC3-I to LC3-II ${ }^{[48,49]}$. Moreover, LC3-II is closely related to the for- 

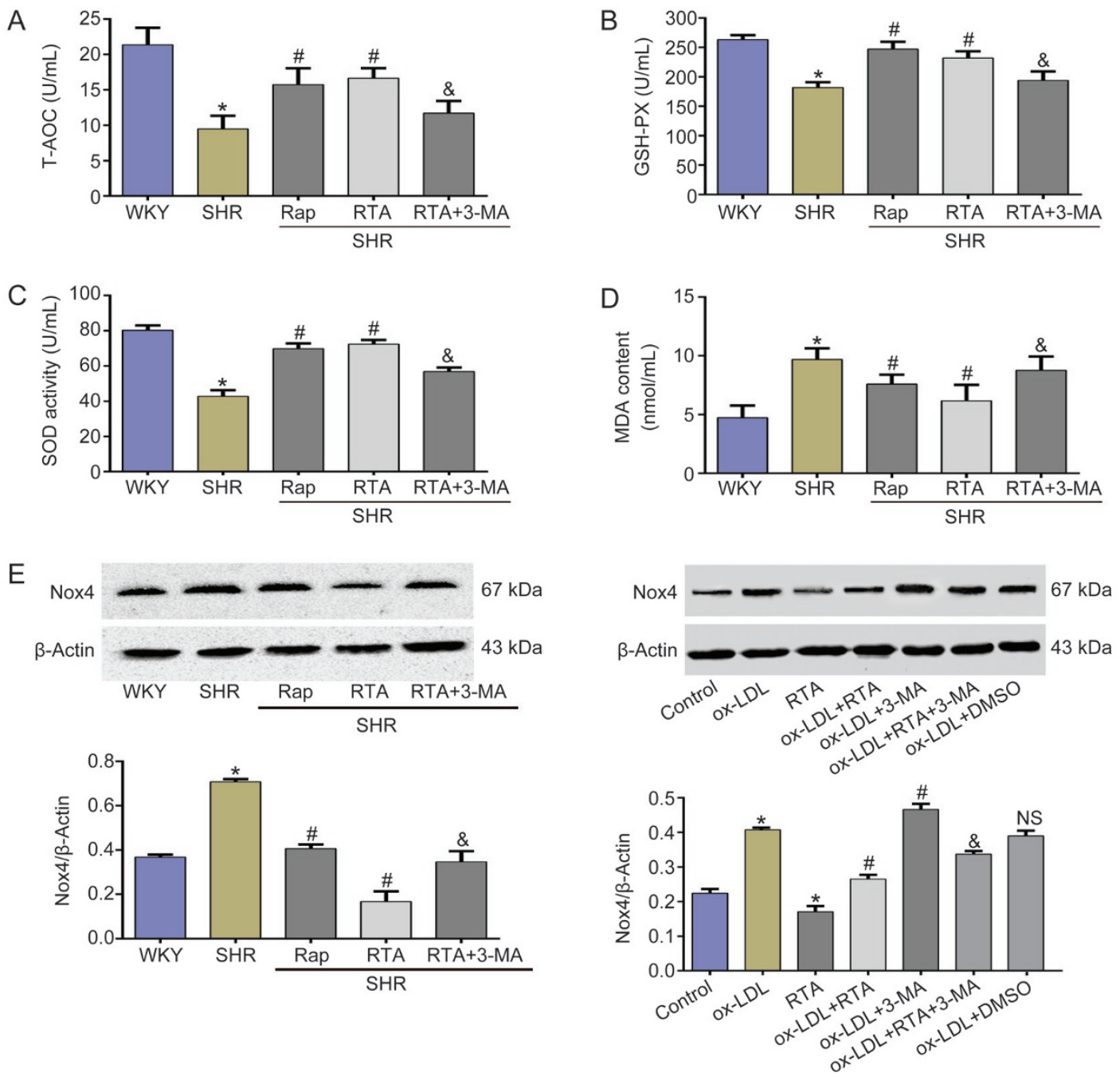

Figure 4. RTA strengthened the antioxidant capacity and alleviated oxidative stress in SHR. (A-D) RTA obviously increased the T-AOC (A), GSH-PX (B), SOD activity (C) and decreased the MDA content (D) in SHR serum. (E) RTA inhibited the expression of Nox4, which was abolished by 3-MA. * $P<0.05$ versus WKY or control group (in vitro), ${ }^{\#} P<0.05$ versus SHR or ox-LDL group (in vitro), ${ }^{\text {NS }} P>0.05$ versus ox-LDL group (in vitro), ${ }^{\&} P<0.05$ vs RTA or ox$\mathrm{LDL}+\mathrm{RTA}$ group (in vitro). Data were pooled as mean $\pm \mathrm{SD}$ (error bars) from three independent experiments.

mation of autophagosomes. Accordingly, the quantification of changes in LC3-II is widely used to evaluate autophagy. Of note, levels of LC3-II should be compared to actin, but not to LC3-I ${ }^{[50]}$. To accurately evaluate autophagy, we also examined the expression of beclin- 1 and $\mathrm{p} 62$. In vitro experiments have shown that autophagy decreases oxidative stress and increases NO production ${ }^{[14]}$. It has been reported that autophagy attenuates endothelial inflammation ${ }^{[26]}$ and stabilizes atherosclerotic plaques $^{[12]}$. Autophagy has also been found to protect the heart from inflammation and cardiac injury induced by hypertension $^{[27]}$. However, the potential role of autophagy in the endothelial vasodilation function has rarely been reported.

It has been demonstrated that oxidative damage induced by ox-LDL results in cell apoptosis. Autophagy is generally considered a protective factor in the vasculature and disease; nevertheless, the role of autophagy in the endothelial cells exposed to ox-LDL is controversial. We first detected the role of autophagy in ox-LDL-treated HUVECs. Our results showed that autophagy was induced in HUVECs of ox-LDL treated for $6 \mathrm{~h}$, as indicated by the increased levels of LC3II and Beclin-1. It has been reported that exposure to ox-LDL for $6 \mathrm{~h}$ also induces autophagy in HUVECs ${ }^{[51]}$, and ox-LDLinduced autophagy has been associated with the endoplasmic reticulum stress and cytosolic calcium deregulation mediated by ox-LDL ${ }^{[52]}$. However, autophagy was impaired by ox-LDL in a time-dependent manner in HUVECs, and a clear effect was observed at $24 \mathrm{~h}$ after ox-LDL exposure. In addition, enhancement of autophagy by Rap decreased apoptosis in oxLDL-treated HUVECs, whereas 3-MA exerted an opposite effect. It has also been reported that enhancing autophagy is a protective mechanism that attenuates oxidative stress and oxLDL-induced HUVEC apoptosis ${ }^{[19]}$.

Our previous studies have confirmed that RTA has a strong effect of decreasing blood pressure and inhibiting inflammatory damage, through a mechanism associated with decreasing the levels of ICAM-1 and VCAM-1 and increasing the 
A
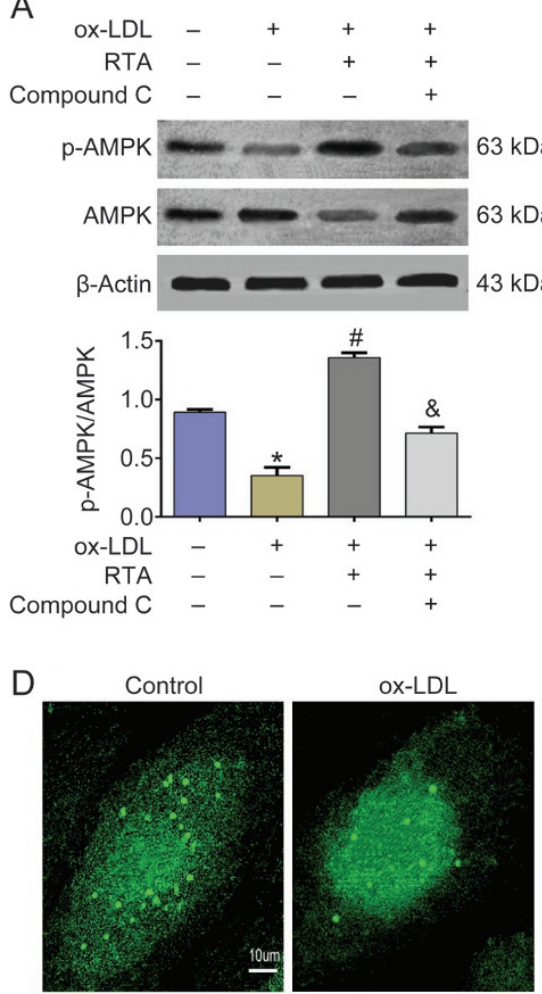

$43 \mathrm{kDa}$
B
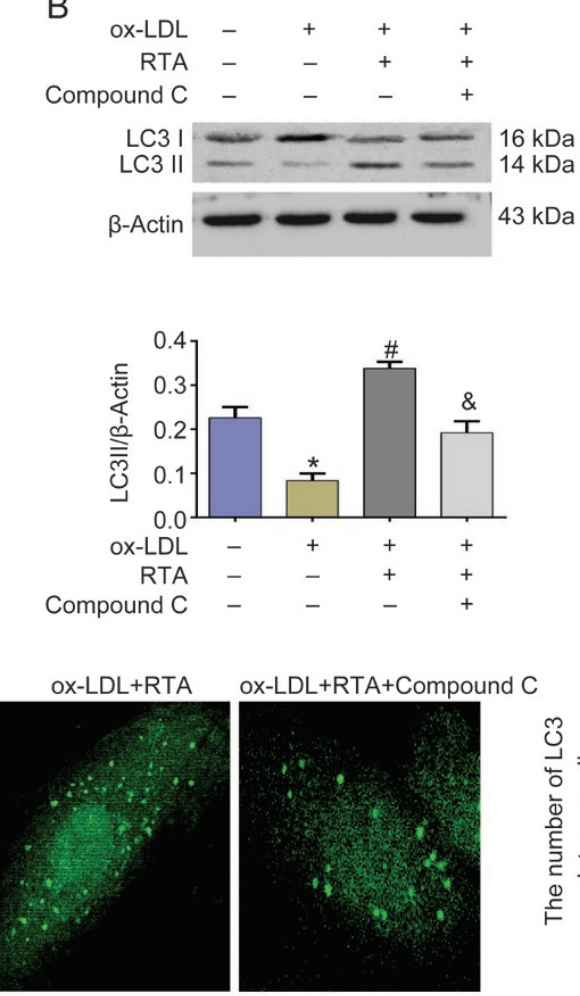
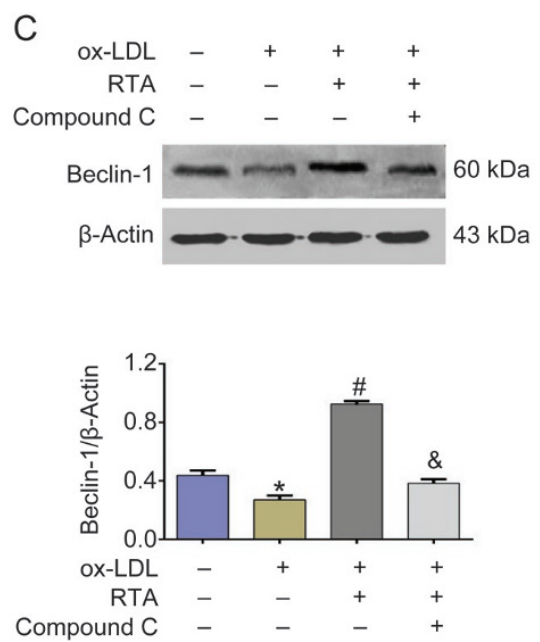

Figure 5. The enhancing of autophagy by RTA was relevant to activating AMPK signaling pathway. (A-D) HUVECs were incubated with either the culture medium (control) or ox-LDL (100 $\mathrm{gg} / \mathrm{mL}$ ) in the absence or presence of RTA and compound C for $24 \mathrm{~h}$. RTA obviously increased p-AMPK (Thr-172) expression (A), LC3-II level (B), beclin-1 level (C) and the number of GFP-LC3 dots (D). Compound C markedly inhibited the effect of RTA. " $P<0.05$ vs control group. ${ }^{\#} P<0.05$ vs ox-LDL group. ${ }^{\circledR} P<0.05$ vs ox-LDL+RTA group. Data were pooled as mean \pm SD (error bars) from three independent experiments.

production of $\mathrm{NO}^{[17,18]}$. Next, we explored the effect of RTA on autophagy in ox-LDL-treated HUVECs. RTA was found to play a novel protective role for HUVECs exposed to ox-LDL manifested by the apoptosis assay results. In addition, RTA also increased SOD activity and decreased MDA content. We therefore concluded that RTA relieves oxidative injury and protects against ox-LDL-induced HUVEC apoptosis. Moreover, TEM showed that the number of autophagosomes was lower in the ox-LDL group (incubated with ox-LDL for $24 \mathrm{~h}$ ) compared with the control group. After culturing with RTA, we also found more autophagosomes and fewer lipid droplets. Moreover, RTA clearly increased LC3II levels, Beclin- 1 expression and the number of GFP-LC3 dots that were counteracted by 3-MA. The effect of RTA in alleviating the oxidative damage was abolished in beclin-1 knockdown HUVECs. These data provide evidence that RTA protects HUVECs against oxidative damage by enhancing autophagy in ox-LDL-treated HUVECs.

Endothelial dysfunction, which is an early feature of atherosclerosis in patients with hypertension, has been found to increase the risk of cardiovascular diseases ${ }^{[28]}$. The involvement of oxidative stress in endothelial dysfunction and atherosclerosis development in hypertensive patients is well established, and ox-LDL plays a primary role ${ }^{[10,2]}$. A previous study has shown that ox-LDL leads to ROS accumulation ${ }^{[19]}$ and activates autophagy, thus attenuating oxidative injury in HUVEC $^{[16,19,20]}$. According to extensive studies, autophagy is a cellular self-protective measure that can attenuate oxidative stress in vascular endothelial cells. Therefore, we speculate that RTA may have a positive role in enhancing autophagy, thus decreasing oxidative stress to improve the endotheliumdependent vasodilation function, which is the central feature of vascular endothelial dysfunction. Further studies are warranted to validate this possibility. We first tested the toxicity reactions, plasma concentration and metabolism of RTA. No toxic reactions were observed in the animals during our experiment (Supplementary Figure 2). The active ingredients of RTA in vivo are mainly rhynchophylline $(43 \%)$ and isorhynchophylline (21\%), according to a previous report ${ }^{[53]}$. The rhynchophylline concentration was $139.673 \mathrm{ng} / \mathrm{mL}$ at 30 $\mathrm{min}$, and it reached a maximum $(1128.241 \mathrm{ng} / \mathrm{mL})$ at $2 \mathrm{~h}$. The rhynchophylline concentration was 9.863 and $0.447 \mathrm{ng} / \mathrm{mL}$ at $12 \mathrm{~h}$ and $24 \mathrm{~h}$, respectively. The isorhynchophylline concentration was $78.074 \mathrm{ng} / \mathrm{mL}$ at $30 \mathrm{~min}$, and it reached a maximum $(459.482 \mathrm{ng} / \mathrm{mL})$ at $2 \mathrm{~h}$. The isorhynchophylline concentration was $6.913 \mathrm{ng} / \mathrm{mL}$ and $1.392 \mathrm{ng} / \mathrm{mL}$ at $12 \mathrm{~h}$ and $24 \mathrm{~h}$, respectively (Supplementary Figure 3). Next, we explored the endothelial vasodilation function by using a vascular ring ten- 
sion test. RTA and Rap markedly improved the endotheliumdependent vasodilation function through enhancing autophagy, whereas inhibition of autophagy exerted the opposite effect. In contrast, there were no significant differences among the groups in the endothelium-independent vasodilation function test.

$\mathrm{NO}$, a vasodilator, plays an important role in the regulation of vascular tone $\mathrm{e}^{[6]}$. An imbalance of $\mathrm{NO}$ and ROS, socalled oxidative stress, may contribute to endothelial dysfunction $^{[21,29]}$. Our results showed that RTA increased the NO content in SHR serum and the expression of eNOS in SHR aorta, whereas these effects were abolished by 3-MA. These data suggested that RTA has a positive role in improving the endothelium-dependent vasodilation function through enhancing autophagy. Another study has also shown that $\mathrm{B}-\mathrm{Fe}_{3} \mathrm{O}_{4} \mathrm{NPs}$ induces autophagy dysfunction in HUVECs and eventually leads to endothelial dysfunction and inflammation ${ }^{[30]}$. In our study, we used a vascular ring tension test to more directly verify the endothelial vasodilation function in vivo, not just the production of NO and eNOS. We also found that RTA improved endothelial vasodilation function, which has also been shown to decrease SHR blood pressure in our previous studies.

In hypertension, increased inactivation of $\mathrm{NO}$ resulting from oxidative stress promotes endothelial dysfunction, and ROS is considered to be a primary factor ${ }^{[31]}$. In addition to directly inhibiting NO activity, ROS decreases the production of NO by inducing eNOS uncoupling ${ }^{[30]}$. Owing to the close relationship between endothelium-dependent vasodilation function and oxidative stress, we analyzed the antioxidant ability and oxidative stress in SHR. We first detected the T-AOC and GSH-PX in SHR serum. RTA clearly increased the levels of T-AOC and GSH-PX in SHR serum, whereas 3-MA abolished this effect. SOD, a major antioxidant enzyme, decreases ROS bioavailability and inhibits oxidative inactivation of $\mathrm{NO}$ in endothelial cells ${ }^{[32,33]}$. Nox4, an isoform of NADPH oxidase, has been identified in vascular walls (particularly VSMCs and endothelial cells) and increases NADPH-dependent ROS production and decreases the bioactivity of $\mathrm{NO}^{[34]}$. Therefore, we speculated that RTA might promote the production of NO by regulating Nox4 expression and SOD activity. RTA rescued the impaired SOD activity and decreased the expression of Nox4 in SHR. As expected, 3-MA blocked the effect of RTA. These data suggested that RTA increases the antioxidant capacity and mitigates oxidative damage through enhancing autophagy. In addition, our data also provided evidence that the upregulation of NO induced by RTA may be related to the decreased Nox4 production and the increased SOD activity. It has been reported that adiponectin stimulates autophagy and increases SOD activity ${ }^{[35]}$. Some recent studies have also demonstrated that Nox4 promotes autophagy ${ }^{[36-38]}$. However, our study indicated that RTA decreased the expression of Nox4 by enhancing autophagy.

We further investigated which signaling pathway mediates the promotion of autophagy by RTA. AMPK activation is required for the induction of autophagy ${ }^{[39,40]}$. Several studies have also suggested that AMPK is a key player in the regulation of lipid metabolism ${ }^{[41]}$, protein synthesis ${ }^{[41]}$, and redox regulation ${ }^{[42,43]}$. Therefore, we analyzed the activation status of AMPK signaling. Our results showed that RTA markedly upregulated p-AMPK (Thr-172) expression, and this effect was abolished by compound C (an inhibitor of AMPK). Moreover, compound $\mathrm{C}$ inhibited the promotion of autophagy by RTA, thus suggesting that promotion of autophagy by RTA results in activation of the AMPK signaling pathway. Several recent studies have shown that activation of AMPK improves endothelial function by increasing eNOS expression and promoting NO production, both of which are associated with activation of the AMPK/eNOS pathway ${ }^{[44-46]}$. In addition, activation of AMPK inhibits Nox4 expression and restrains Nox-derived ROS overproduction ${ }^{[4]}$. Therefore, we speculated that activation of AMPK by RTA might be involved in improving endothelial vasodilation function by promoting eNOS expression and NO release. However, further experiments are needed to verify this possibility.

However, several limitations of our study should be noted. Because we determined the optimum culturing concentration of ox-LDL in our previous study, experiments under other conditions of ox-LDL were not performed. In this study, we primarily observed the effect of RTA on autophagy and endothelial vasodilation function. We will further investigate how RTA activates AMPK signaling in our next study. In addition, more studies are needed to elucidate the potential mechanisms of alleviating the oxidative stress.

\section{Conclusions}

RTA has a positive role in enhancing autophagy impaired by ox-LDL in HUVECs, thus decreasing oxidative stress and improving endothelium-dependent vasodilation function; AMPK signaling is involved in the promotion of autophagy by RTA. Hypertension treatment is not meant only to control blood pressure; target-organ protection is also important. Our findings provide new insights into the treatment of hypertension and the development of antihypertensive drugs.

\section{Acknowledgements}

This study was supported by the National Natural Science Foundation of China (№ 81273700, 81473653)

\section{Author contribution}

Chao LI and Feng JIANG performed all the experiments. Chao LI and Yun-lun LI wrote the main manuscript. Yuehua JIANG and Wen-qing YANG prepared Figures 1-3. Jie SHENG prepared Figure 4. Wen-juan XU prepared Figure 5. Qing-jun ZHU participated in animal model design. All authors reviewed the manuscript.

\section{References}

1 Tanito M, Nakamura H, Kwon YW, Teratani A, Masutani H, Shioji K, et al. Enhanced oxidative stress and impaired thioredoxin expression in spontaneously hypertensive rats. Antioxid Redox Signal 2004; 6: 89-97. 
2 Kitts DD, Yuan YV, Godin DV. Plasma and lipoprotein lipid composition and hepatic antioxidant status in spontaneously hypertensive (SHR) and normotensive (WKY) rats. Can J Physiol Pharmacol 1998; 76 : 202-9.

3 Redón J, Oliva MR, Tormos C, Giner V, Chaves J, Iradi A, et al. Antioxidant activities and oxidative stress byproducts in human hypertension. Hypertension 2003; 41: 1096-101.

4 Touyz RM. Reactive oxygen species, vascular oxidative stress, and redox signaling in hypertension: what is the clinical significance? Hypertension 2004; 44: 248-52.

5 Perrotta I, Aquila S. The role of oxidative stress and autophagy in atherosclerosis. Oxid Med Cell Longev 2015. doi: 10.1155/2015/130315.

6 Higashi Y, Noma K, Yoshizumi M, Kihara Y. Endothelial function and oxidative stress in cardiovascular diseases. Circ J 2009; 73: 411-8.

7 Li H, Horke S, Förstermann U. Oxidative stress in vascular disease and its pharmacological prevention. Trends Pharmacol Sci 2013; 34: 313-9.

8 Forstermann U. Nitric oxide and oxidative stress in vascular disease. Pflugers Arch 2010; 459: 923-39.

9 Zou MH, Cohen R, Ullrich V. Peroxynitrite and vascular endothelial dysfunction in diabetes mellitus. Endothelium 2004; 11: 89-97.

10 Mitra S, Goyal T, Mehta JL. Oxidized LDL, LOX-1 and atherosclerosis. Cardiovasc Drug Ther 2011; 25: 419-29.

11 Tsounis D, Bouras G, Giannopoulos G, Papadimitriou C, Alexopoulos D, Deftereos S. Inflammation markers in essential hypertension. Med Chem 2014; 10: 672-81.

12 Schrijvers DM, De Meyer GR, Martinet W. Autophagy in atherosclerosis: a potential drug target for plaque stabilization. Arteriosclerosis Thromb Vasc Biol 2011; 31: 2787-91.

13 De Meyer GR, Martinet W. Autophagy in the cardiovascular system. Biochim Biophys Acta 2009; 1793: 1485-95.

14 LaRocca TJ, Henson GD, Thorburn A, Sindler AL, Pierce GL, Seals DR. Translational evidence that impaired autophagy contributes to arterial ageing. J Physiol 2012; 590: 3305-16.

$15 \mathrm{Lin} \mathrm{HH}$. In vitro and in vivo atheroprotective effects of gossypetin against endothelial cell injury by induction of autophagy. Chem Res Toxicol 2015; 28: 202-15.

16 Jin X, Chen M, Yi L, Chang H, Zhang T, Wang L, et al. Delphinidin-3glucoside protects human umbilical vein endothelial cells against oxidized low-density lipoprotein-induced injury by autophagy upregulation via the AMPK/SIRT1 signaling pathway. Mol Nutr Food Res 2014; 58: 1941-51.

17 Yang JG, Li YL, Zhou HL. Protective effect of total alkaloids from Uncariae Ramulus cum Uncis combined with Raphani Semen on vascular endothelial cell injured by hypertension. Chin Tradit Patent Med 2013; 35: 889-93.

18 Jiao HC, Li YL, Jiang YH, Qi DM, Han T, Jiang HQ. The effect and mechanism of component compatibility of rhynchophylla total alkaloids and sinapine cyanide sulfonate on change of vascular endothelial cells. Chin J Arterioscler 2013; 21: 397-403.

19 Luo X, Yang Z, Zheng S, Cao Y, Wu Y. Sirt3 activation attenuated oxidized low-density lipoprotein-induced human umbilical vein endothelial cells' apoptosis by sustaining autophagy. Cell Biol Int 2014. doi: 10.1002/cbin.10291.

20 Guo H, Chen Y, Liao L, Wu W. Resveratrol protects HUVECs from oxidized-LDL induced oxidative damage by autophagy upregulation via the AMPK/SIRT1 pathway. Cardiovasc Drugs Ther 2013; 27: 189-98.

21 Higashi Y, Kihara Y, Noma K. Endothelial dysfunction and hypertension in aging. Hypertens Res 2012; 35: 1039-47.

22 Perrotta I, Aquila S. The role of oxidative stress and autophagy in atherosclerosis. Oxid Med Cell Longev 2015. doi: 10.1155/2015/130315.
23 Briones AM, Touyz RM. Oxidative stress and hypertension: current concepts. Curr Hypertens Rep 2010; 12: 135-42.

24 De Meyer GR, Grootaert MO, Michiels CF. Autophagy in the cardiovascular system. Biochim Biophys Acta 2015; 116: 468-79.

25 Maiuri MC, Grassia G, Platt AM, Carnuccio R, lalenti A, Maffia P. Macrophage autophagy in atherosclerosis. Mediators Inflamm 2013. doi: 10.1155/2013/584715.

26 Chen ML, Yi L, Jin X, Liang XY, Zhou Y, Zhang T, et al. Resveratrol attenuates vascular endothelial inflammation by inducing autophagy through the cAMP signaling pathway. Autophagy 2013; 9: 2033-45.

27 Zhao W, Li Y, Jia L, Pan L, Li H, Du J. Atg5 deficiency-mediated mitophagy aggravates cardiac inflammation and injury in response to angiotensin II. Free Radic Biol Med 2014; 69: 108-15.

28 Higashi Y, Kihara Y, Noma K. Endothelial dysfunction and hypertension in aging. Hypertens Res 2012; 35: 1039-47.

29 Siti HN, Kamisah Y, Kamsiah J. The role of oxidative stress, antioxidants and vascular inflammation in cardiovascular disease (a review). Vasc Pharmacol 2015; 71: 40-56.

30 Zhang L, Wang X, Miao Y, Chen Z, Qiang P, Cui L, et al. Magnetic ferroferric oxide nanoparticles induce vascular endothelial cell dysfunction and inflammation by disturbing autophagy. J Hazard Mater 2016; 304: 186-95.

31 Montezano AC, Dulak-Lis M, Tsiropoulou S, Harvey A, Briones AM, Touyz RM. Oxidative stress and human hypertension: vascular mechanisms, biomarkers, and novel therapies. Can J Cardiol 2015; 31: 631-41.

32 Maksimenko AV. Experimental antioxidant biotherapy for protection of the vascular wall by modified forms of superoxide dismutase and catalase. Curr Pharm Design 2005; 11: 2007-16.

33 Fukai T, Ushio-Fukai M. Superoxide dismutases: role in redox signaling, vascular function, and diseases. Antioxid Redox Signal 2011; 15: 1583-606.

34 Konior A, Schramm A, Czesnikiewicz-Guzik M, Guzik TJ. NADPH oxidases in vascular pathology. Antioxid Redox Signal 2014; 20 : 2794-814.

35 Liu Y, Palanivel R, Rai E, Park M, Gabor TV, Scheid MP, et al. Adiponectin stimulates autophagy and reduces oxidative stress to enhance insulin sensitivity during high fat diet feeding in mice. Diabetes 2015; 64: 36-48.

36 Sciarretta S, Yee D, Ammann P, Nagarajan N, Volpe M, Frati G, et al. Role of NADPH oxidase in the regulation of autophagy in cardiomyocytes. Clin Sci 2015; 128: 387-403.

37 He C, Zhu H, Zhang W, Okon I, Wang Q, Li H, et al. 7-Ketocholesterol induces autophagy in vascular smooth muscle cells through Nox4 and Atg4B. Am J Pathol 2013; 183: 626-37.

38 Wu RF, Ma Z, Liu Z, Terada LS. Nox4-Derived H2O2 mediates endoplasmic reticulum signaling through local ras activation. Mol Cell Biol 2010; 30: 3553-68.

39 Park SY, Lee HR, Lee WS, Shin HK, Kim HY, Hong KW, et al. Cilostazol modulates autophagic degradation of beta-amyloid peptide via SIRT1Coupled LKB1/AMPKalpha signaling in neuronal cells. PLoS One 2016. doi: 10.1371/journal.pone.0160620.

40 Jeon SM. Regulation and function of AMPK in physiology and diseases. Exp Mol Med 2016; 48: e245.

41 Habets DD, Coumans WA, El Hasnaoui M, Zarrinpashneh E, Bertrand $\mathrm{L}$, Viollet $\mathrm{B}$, et al. Crucial role for LKB1 to AMPKalpha2 axis in the regulation of $C D 36$-mediated long-chain fatty acid uptake into cardiomyocytes. Biochim Biophys Acta 2009; 1791: 212-9.

42 Hung $\mathrm{CH}$, Chan SH, Chu PM, Lin HC, Tsai KL. Metformin regulates oxLDL-facilitated endothelial dysfunction by modulation of SIRT1 through repressing LOX-1-modulated oxidative signaling. Oncotarget 2016; 7: 
10773-87.

43 Jeon SM, Chandel NS, Hay N. AMPK regulates NADPH homeostasis to promote tumour cell survival during energy stress. Nature 2012; 485: 661-5.

44 Li N, Zhao Y, Yue Y, Chen L, Yao Z, Niu W. Liraglutide ameliorates palmitate-induced endothelial dysfunction through activating AMPK and reversing leptin resistance. Biochem Biophys Res Commun 2016; 478: 46-52.

45 Choy KW, Mustafa MR, Lau YS, Liu J, Murugan D, Lau CW, et al. Paeonol protects against endoplasmic reticulum stress-induced endothelial dysfunction via AMPK/PPARdelta signaling pathway. Biochem Pharmacol 2016; 116: 51-62.

46 Xing SS, Yang XY, Zheng T, Li WJ, Wu D, Chi JY, et al. Salidroside improves endothelial function and alleviates atherosclerosis by activating a mitochondria-related AMPK/PI3K/Akt/eNOS pathway. Vasc Pharmacol 2015; 72: 141-52.

47 He T, Xiong J, Nie L, Yu Y, Guan X, Xu X, et al. Resveratrol inhibits renal interstitial fibrosis in diabetic nephropathy by regulating AMPK/NOX4/ ROS pathway. J Mol Med 2016. doi:10.1007/s00109-016-1451-y.

48 Cai Q, Lu L, Tian JH, Zhu Y-B, Qiao H, Sheng ZH. Snapin-regulated late endosomal transport is critical for efficient autophagylysosomal function in neurons. Neuron 2010; 68: 73-86.

49 Castino R, Fiorentino I, Cagnin M, Giovia A, Isidoro C. Chelation of lysosomal iron protects dopaminergic SH-SY5Y neuroblastoma cells from hydrogen peroxide toxicity by precluding autophagy and Akt dephosphorylation. Toxicol Sci 2011; 123: 523-41.

50 Klionsky DJ, Abdelmohsen K, Abe A, Abedin MJ, Abeliovich H, Acevedo Arozena $A$, et al. Guidelines for the use and interpretation of assays for monitoring autophagy (3rd edition). Autophagy 2016; 12: 1-222.

51 Zhang YL, Cao YJ, Zhang X, Liu HH, Tong T, Xiao GD, et al. The autophagylysosome pathway: a novel mechanism involved in the processing of oxidized LDL in human vascular endothelial cells. Biochem Biophys Res Commun 2010; 39: 377-82.

52 Muller C, Salvayre R, Nègre-Salvayre A, Vindis C. Oxidized LDLs trigger endoplasmic reticulum stress and autophagy: prevention by HDLs. Autophagy 2011; 7: 541-3.

53 Shi JS, Yu JX, Chen XP, Xu RX. Pharmacological actions of Uncaria alkaloids, rhynchophylline and isorhynchophylline. Acta Pharmacol Sin 2003; 24: 97-101. 